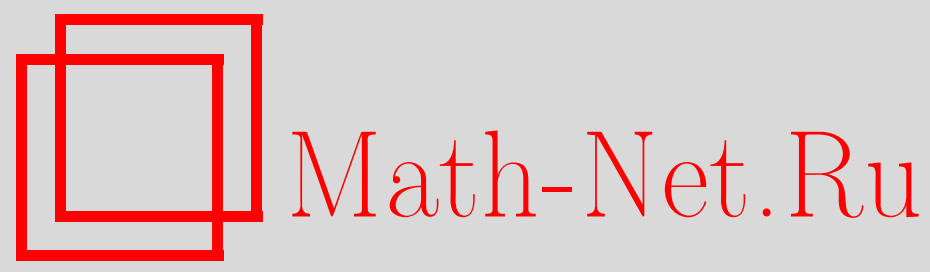

С. А. Зеленова, Коммутативные подалгебры квантовых алгебр, Матем. заметки, 2004, том 75, выпуск 2, 208-221

DOI: https://doi.org/10.4213/mzm24

Использование Общероссийского математического портала Math-Net.Ru подразумевает, что вы прочитали и согласны с пользовательским соглашением http://www . mathnet.ru/rus/agreement

Параметры загрузки:

IP: 3.89 .197 .203

26 апреля 2023 г., 09:13:45 


\title{
КОММУТАТИВНЫЕ ПОДАЛГЕБРЫ КВАНТОВЫХ АЛГЕБР
}

\author{
С. А. Зеленова
}

В настоящей работе доказывается общее утверждение о том, что в любой ассоциативной алгебре $\mathscr{A}$ без делителей нуля, обладающей нормой и согласованной с ней полунормой, степень трансцендентности произвольной коммутативной подалгебры не превьшает максимального количества независимых попарно псевдокоммутирующих элементов некоторого базиса алгебры $\mathscr{A}$. Автором показано, что в качестве подобной алгебры $\mathscr{A}$ могут быть рассмотрены алгебры квантовых лорановских многочленов, квантовые аналоги алгебры Вейля, а также некоторые универсальные кодействующие алгебры. В случае алгебры $\mathscr{L}$ квантовых лорановских многочленов доказано, что степень трансцендентности максимальной коммутативной подалгебры алгебры $\mathscr{L}$ совпадает с максимальным количеством независимых попарно коммутирующих элементов мономиального базиса алгебры $\mathscr{L}$.

Библиографиия: 8 названий.

\section{Введение}

В настоящей работе изучаются коммутативные подалгебры некоторых квантовых алгебр.

Напомним, что в работе [1] показано, что в теле частных $k_{q}(X, Y)$ алгебры квантовых многочленов $\Lambda=k_{q}[X, Y]$ централизатор любого элемента, отличного от константы, коммутативен и потому является максимальньм подполем в $k_{q}(X, Y)$. Идея доказательства восходит к [2]. В статье [3] эта ситуация обобщена на случай трех и более переменных в предположении, что поле $k$ имеет нулевую характеристику. В работе [4] ограничение на характеристику снимается и предлагается более простое доказательство. Кроме того, в той же работепоказано, что в алгебре квантовых многочленов размерность Крулля любой коммутативной подалгебры не вьше 1, и построена коммутативная подалгебра, не являющаяся чисто трансцендентным расширением $k$.

В работах [1], [3] поставлен вопрос об описании подполей в теле частньх алгебры общих квантовых многочленов.

В настоящей работе доказьвается общее утверждение о том, что в любой ассоциативной алгебре $\mathscr{A}$ без делителей нуля, обладающей нормой и согласованной с ней полунормой, степень трансцендентности произвольной коммутативной подалгебры не превышает максимального количества независимых попарно псевдокоммутирующих элементов некоторого базиса алгебры $\mathscr{A}$.

Автором показано, что в качестве подобной алгебры $\mathscr{A}$ могут быть рассмотрены алгебры квантовых лорановских многочленов, квантовые аналоги алгебры Вейля (см. [5]), а также некоторые универсальные кодействующие алгебры (см. [6]). 
В случае алгебры $\mathscr{L}$ квантовых лорановских многочленов доказано, что степень трансцендентности максимальной коммутативной подалгебры алгебры $\mathscr{L}$ совпадает с максимальньм количеством независимых попарно коммутирующих элементов мономиального базиса алгебры $\mathscr{L}$.

Рассматриваемые в настоящей работе алгебрыпредставляют большой интерес с точки зрения некоммутативной алгебраической геометрии и теории квантовых групп. Общие вопросы, связанные с квантовыми алгебрами, изучаются в монографии [7].

Автор выражает искреннюю благодарность В. А. Артамонову за постановку задачи и руководство работой.

\section{1. Предварительные сведения}

Вначале дадим несколько вспомогательных определений.

Через $\sigma(\mathbf{v})$ будем обозначать сумму элементов целочисленного кортежа $\mathbf{v}=$ $\left(v_{1}, \ldots, v_{n}\right) \in \mathbb{Z}^{n}$.

ОПРЕДЕЛЕниЕ 1.1. Рассмотрим отношение порядка $\succ$ на множестве $\mathbb{Z}^{n}$. Положим

$$
\mathbf{u}=\left(u_{1}, \ldots, u_{n}\right) \succ\left(v_{1}, \ldots, v_{n}\right)=\mathbf{v}, \quad \mathbf{u}, \mathbf{v} \in \mathbb{Z}^{n}
$$

если выполнено одно из условий

1) $\sigma(\mathbf{u})>\sigma(\mathbf{v})$;

2) $\sigma(\mathbf{u})=\sigma(\mathbf{v})$ и существует такое $j \in\{1, \ldots, n\}$, что $u_{i}=v_{i}$ для всех $i=1, \ldots, j-$ 1 и $u_{j}>v_{j}$.

Иными словами, кортежи из $\mathbb{Z}^{n}$ сначала сравниваются по сумме своих координат, а в случае равенства таких сумм порядок $\succ$ совпадает с лексикографическим.

Лемма 1.2. Введенный порядок обладает следующими свойствами:

1) $\mathbf{u}+\mathbf{t} \succ \mathbf{u}$, еслu $\mathbf{t}=\left(t_{1}, \ldots, t_{n}\right) \in \mathbb{N}_{0}^{n}$

2) $\mathbf{u}+\mathbf{t} \succ \mathbf{v}+\mathbf{t}$, если $\mathbf{u} \succ \mathbf{v} \partial$ дя всех $\mathbf{t} \in \mathbb{Z}^{n}$;

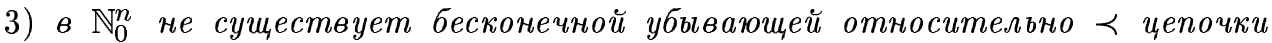
(здесь $\mathbb{N}_{0}$ обозначает множество неотричательных иельх чисел);

4) зафиксируем әлемент $\mathbf{d} \in \mathbb{N}_{0}^{n} ;$ пусть подмножество $M \subset \mathbb{N}_{0}^{n}$ таково, что $\mathbf{v} \preceq \mathbf{d} \partial$ ля всех $\mathbf{v} \in M ;$ тогда $M$ конечно.

ДоКАЗАТЕЛЬСтво. Свойства 1) и 2) проверяются непосредственно.

3) Элементов $\mathbb{N}_{0}^{n}$ с одинаковой фиксированной суммой координат конечное число, поэтому любой элемент $\mathbb{N}_{0}^{n}$ имеет конечное число меньших.

4) Будем доказывать искомое утверждение индукцией по $n$. При $n=1$ оно очевидно.

Пусть наше утверждение доказано для всех натуральных чисел меньших $n$. Фиксируем первую координату $v_{1}$ кортежа $\mathbf{v}$. Поскольку $\mathbf{v} \preceq \mathbf{d}$, то $\sigma(\mathbf{v}) \preceq \sigma(\mathbf{d})$. Поэтому $\left(v_{2}, \ldots, v_{n}\right) \prec\left(d_{1}+d_{2}+1, d_{3}, \ldots, d_{n}\right)$. По предположению индукции таких кортежей $\left(v_{2}, \ldots, v_{n}\right)$ конечное число. Теперь заметим, что на первую координату кортежа $\mathbf{v}$ накладьвается условие $0 \leqslant v_{1} \leqslant \sigma(\mathbf{v}) \leqslant \sigma(\mathbf{d})$, т.е. вариантов для $v_{1}$ также конечное число. 
ОПРЕДЕЛЕНИЕ 1.3. Абсолютным значением кортежа $\mathbf{z}=\left(z_{1}, \ldots, z_{n}\right) \in \mathbb{Z}^{n}$ назовем кортеж из абсолютных значений элементов $\mathbf{z}$ :

$$
\left|\left(z_{1}, \ldots, z_{n}\right)\right|=\left(\left|z_{1}\right|, \ldots,\left|z_{n}\right|\right)
$$

УТВЕРЖДЕНИЕ 1.4. Для произвольных кортежей $\mathbf{u} u \mathbf{v}$ выполняется неравенство $|\mathbf{u}+\mathbf{v}| \preceq|\mathbf{u}|+|\mathbf{v}|$.

ДокАЗАТЕльство. Для произвольных вещественных чисел $x, y$ имеем $|x+y| \leqslant|x|+$ $|y|$. Поэтому

$$
\sigma(|\mathbf{u}+\mathbf{v}|)=\sum_{i=1}^{n}\left|u_{i}+v_{i}\right| \leqslant \sum_{i=1}^{n}\left|u_{i}\right|+\left|v_{i}\right|=\sigma(|\mathbf{u}|+|\mathbf{v}|)
$$

Равенство вьполняется только тогда, когда для всех $i=1, \ldots, n$ вьполнены равенства $\left|u_{i}+v_{i}\right|=\left|u_{i}\right|+\left|v_{i}\right|$, т.е. когда кортежи $|\mathbf{u}+\mathbf{v}|$ и $|\mathbf{u}|+|\mathbf{v}|$ равны.

Теперь утверждение очевидно.

ЛЕмма 1.5. Зафиксируем әлемент $\mathbf{d} \in \mathbb{N}_{0}^{n}$. Пусть подмножество $M \subset \mathbb{Z}^{n}$ таково, что $|\mathbf{v}| \preceq \mathbf{d}$ для всех $\mathbf{v} \in M$. Тогда $M$ конечно.

ДокАЗАТЕЛЬСтво. Утверждение леммы следует из пункта 4) леммы 1.2 и того, что для заданного $\mathbf{t} \in \mathbb{N}_{0}^{n}$ количество элементов $\mathbf{v} \in \mathbb{Z}^{n}$ таких, что $|\mathbf{v}|=\mathbf{t}$, конечно.

\section{2. Теорема об алгебраической зависимости}

Пусть $k$ - основное поле и пусть $\mathscr{A}$ - ассоциативная алгебра без делителей нуля над этим полем.

Предположим, что существует отображение $\eta$ алгебры $\mathscr{A}$ в множество $\mathbb{Z}^{n}$, удовлетворяющее следующим соотношениям:

N1) $\eta(\alpha f)=\eta(f)$ для всех ненулевых $f \in \mathscr{A}_{\text {и }} \alpha \in k^{*}$;

N2) $\eta(f+g) \preceq \max \{\eta(f), \eta(g)\}$ для всех ненулевых $f, g \in \mathscr{A}$;

N3) $\eta(f g)=\eta(f)+\eta(g)$ для всех ненулевых $f, g \in \mathscr{A}$.

Кроме того, предположим, что существует базис $\mathfrak{B}=\left\{b_{i}\right\}_{i \in \mathscr{I}}$ алгебры $\mathscr{A}$, рассматриваемой как векторное пространство, такой, что вьполнены следующие условия:

N4) $\eta\left(b_{i}\right) \neq \eta\left(b_{j}\right)$ для любых $b_{i} \neq b_{j}, b_{i}, b_{j} \in \mathfrak{B}$;

$\mathrm{N} 5)$ если $f=\sum_{s=1}^{r_{f}} \alpha_{s} b_{s}$ разложение произвольного элемента $f \in \mathscr{A}$ по базису $\mathfrak{B}$, то

$$
\eta(f)=\max _{s=1, \ldots, r_{f}} \eta\left(b_{s}\right)
$$

здесь максимум берется относительно порядка $\prec$.

ОПРЕДЕЛЕНИЕ 2.1. Будем назьвать $\eta(f)$ нормой элемента $f$.

Из свойств N1)-N5) нормы сразу вытекает следующее простое утверждение.

ЛЕмма 2.2. Если $\eta(f)=\eta(g)=\mathbf{t} \in \mathbb{Z}^{n}$, то существуют ненулевые $\alpha, \beta \in k$ такие, что $\eta(\alpha f+\beta g) \prec \mathbf{t}$. 
ДокАЗАТЕльСтво. В силу свойства N4) разложение произвольного элемента $h$ алгебры $\mathscr{A}$ по базису $\mathfrak{B}$ содержит единственньй элемент базиса, норма которого совпадает с нормой самого $h$. Поскольку нормы $f$ и $g$ равны, то такой элемент базиса у них общий. Обозначим его через $b$. Тогда $f=\beta b+f^{\prime}, g=\alpha b+g^{\prime}$, где $\eta\left(f^{\prime}\right) \prec \eta(f)=\mathbf{t}$, $\eta\left(g^{\prime}\right) \prec \eta(g)=\mathbf{t}$, а $\alpha, \beta$ - ненулевые элементы поля $k$. Для линейной комбинации $\alpha f-\beta g$ по свойству N2) имеем

$$
\eta(\alpha f-\beta g)=\eta\left(\alpha \beta b+f^{\prime}-\beta \alpha b+g^{\prime}\right)=\eta\left(f^{\prime}-g^{\prime}\right) \preceq \max \left\{\eta\left(f^{\prime}\right), \eta\left(g^{\prime}\right)\right\} \prec \mathbf{t},
$$

что и требовалось.

Пусть $f$ - произвольньй ненулевой элемент $\mathscr{A}$, и пусть $f=\sum_{s=1}^{r_{f}} \alpha_{s} b_{s}$ - его разложение в базисе $\mathfrak{B}$. Определим отображение $\rho$ алгебры $\mathscr{A}$ в множество $\mathbb{N}_{0}^{n}$, связанное с нормой $\eta$. По определению

$$
\rho(f)=\max _{s=1, \ldots, r_{f}}\left|\eta\left(b_{s}\right)\right| .
$$

В дальнейшем нам понадобятся некоторые свойства этого отображения.

Лемма 2.3. Отображсение $\rho$ обладает следующими свойствами:

1) $\rho(\alpha f)=\rho(f)$ для всех ненулевых $f \in \mathscr{A} u \alpha \in k^{*}$;

2) $\rho(f+g) \preceq \max \{\rho(f), \rho(g)\}$ для всех ненулевых $f, g \in \mathscr{A}$, причем строгое неравенство возможно только при $\rho(f)=\rho(g)$;

3) если $f=\sum_{s=1}^{r_{f}} \alpha_{s} b_{s}$ в базисе $\mathfrak{B}$, mo

$$
\rho(f)=\max _{s=1, \ldots, r_{f}} \rho\left(b_{s}\right)
$$

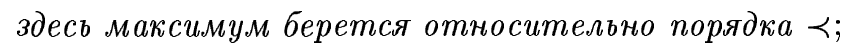

4) $|\eta(f)| \preceq \rho(f)$ для любого ненулевого $f \in \mathscr{A}$;

5) если $M-$ подмножество алгебры $\mathscr{A}$ и $\rho(f) \preceq \mathbf{d}$ для всех $f \in M$ и фиксированного $\mathbf{d} \in \mathbb{N}_{0}^{n}$, то множсество норм әлементов $M$ конечно;

6) множество әлементов базиса $\mathfrak{B}$ с фиксированной полунормой конечно.

ДокАЗАТЕЛЬСтво. Свойства 1), 2) непосредственно следуют из определения.

3) Из определения отображения $\rho$ следует, что $\rho(b)=|\eta(b)|$ для любого $b \in \mathfrak{B}$. Отсюда $\rho(f)=\max _{s=1, \ldots, r_{f}}\left|\eta\left(b_{s}\right)\right|=\max _{s=1, \ldots, r_{f}} \rho\left(b_{s}\right)$.

4) Имеем $|\eta(f)|=\left|\max _{s=1, \ldots, r_{f}} \eta\left(b_{s}\right)\right| \preceq \max _{s=1, \ldots, r_{f}}\left|\eta\left(b_{s}\right)\right|=\rho(f)$.

5) Из 4) получаем $|\eta(f)| \preceq \rho(f) \preceq \mathbf{d}$ для всех $f \in M$. Для доказательства утверждения теперь достаточно использовать лемму 1.5 .

6) Утверждение следует из 5) и свойства N4) нормы.

ОПРЕДЕЛЕНИЕ 2.4. Будем назьвать отображение $\rho$ полунормой, согласованной с нормой $\eta$, если оно удовлетворяет свойству

$$
\rho(f g) \preceq \rho(f)+\rho(g) \quad \text { для всех ненулевьх } f, g \in \mathscr{A} \text {. }
$$

В дальнейшем будем предполагать, что $\mathscr{A}$ - некоторая ассоциативная алгебра без делителей нуля, обладающая нормой $\eta$ и согласованной с ней полунормой $\rho$.

Рассмотрим произвольную подалгебру $\mathscr{P}$ алгебры $\mathscr{A}$. 
Разобьем $\mathscr{P}$ напопарно непересекающиеся подмножества следуюшим образом. Пусть $\mathbf{t} \in \mathbb{N}_{0}^{n}$. Через $\mathscr{P} \mathbf{t}$ обозначим множество всех тех элементов $\mathscr{P}$, полунорма которых равна t.

Обозначим через $\nu_{\mathbf{t}}(p)$ проекцию элемента $p$ на конечномерное векторное пространство, порожденное элементами $\{b \in \mathfrak{B} \mid \rho(b)=\mathbf{t}\}$.

Поскольку образ проекции $\nu_{\mathbf{t}}(\mathscr{P})$ является конечномерньм векторным пространством, в нем можно выбрать конечньй базис.

ОПРЕДЕЛЕНИЕ 2.5. Пусть $\left\{e_{i}\right\}_{i=1}^{r}-$ произвольный базис пространства $\nu_{\mathbf{t}}(\mathscr{P})$. Всякую систему $\left\{h_{i}\right\}_{i=1}^{r}$, где $h_{i} \in \nu_{\mathbf{t}}^{-1}\left(e_{i}\right)$, будем называть базой подмножества $\mathscr{P}_{\mathbf{t}}$.

УТВЕРЖДЕНИЕ 2.6. Существует база $\left\{h_{i}\right\}_{i=1}^{r}$ множества $\mathscr{P}_{\mathbf{t}}$, обладающая следующими свойствами:

(*) нормы $h_{i}$ различны;

$(* *) \eta\left(h_{i}\right) \preceq \eta(h)$ для всякого $h \in \nu_{\mathbf{t}}^{-1}\left(\nu_{\mathbf{t}}\left(h_{i}\right)\right)$.

ДокАЗАТЕЛЬСтво. Пусть $\left\{h_{i}^{0}\right\}_{i=1}^{r}-$ произвольная база множества $\mathscr{P} \mathbf{t}$. В силу утверждения 5 леммы 2.3 множество норм элементов $\nu_{\mathbf{t}}^{-1}\left(\nu_{\mathbf{t}}\left(h_{i}^{0}\right)\right)$ конечно, т.е. мы можем выбрать в $\nu_{\mathbf{t}}^{-1}\left(\nu_{\mathbf{t}}\left(h_{i}^{0}\right)\right)$ элемент с минимальной нормой. Поэтому мы можем считать, что база $\left\{h_{i}^{0}\right\}_{i=1}^{r}$ удовлетворяет свойству $(* *)$.

Пусть эта база упорядочена по возрастанию норм, т.е. $\eta\left(h_{i}^{0}\right) \preceq \eta\left(h_{i+1}^{0}\right)$ для всех $i=1, \ldots, r-1$.

Предположим, что $\eta\left(h_{r-1}^{0}\right)=\eta\left(h_{r}^{0}\right)$. Тогда по лемме 2.2 найдутся такие ненулевые коэффищиенты $\beta_{1}, \beta_{2} \in k$, что

$$
\eta\left(\beta_{1} h_{r-1}^{0}+\beta_{2} h_{r}^{0}\right) \prec \eta\left(h_{r}^{0}\right)
$$

Положим

$$
h_{r-1}^{\prime}=\beta_{1} h_{r-1}^{0}+\beta_{2} h_{r}^{0}, \quad h_{i}^{\prime}=h_{i}^{0} \quad \text { при } i \neq r-1 .
$$

Очевидно, система $\left\{h_{i}^{\prime}\right\}_{i=1}^{r}$ останется базой.

Далее, как и ранее, мы можем преобразовать эту базу в базу, удовлетворяющую свойству $(* *)$. Переупорядочим элементы новой базы так, чтобы нормы их не убывали при возрастании индексов. Повторяя предыдущие рассуждения, приходим к системе $\left\{h_{i}\right\}_{i=1}^{r}$, в которой $\eta\left(h_{r-1}\right) \prec \eta\left(h_{r}\right)$.

Теперь перейдем к системе $\left\{h_{i}\right\}_{i=1}^{r-1}$. Если $\eta\left(h_{r-2}\right) \prec \eta\left(h_{r-1}\right)$, то переходим к системе $\left\{h_{i}\right\}_{i=1}^{r-2}$. Если же нормы $h_{r-1}$ и $h_{r-2}$ равны, то, делая необходимые замены элементов, приходим к системе, в которой $\eta\left(h_{r-2}\right) \prec \eta\left(h_{r-1}\right)$, и т.д.

Для каждого $\mathbf{t} \in \mathbb{N}_{0}^{n}$ зафиксируем базу $\left\{h_{i}^{\mathbf{t}}\right\}_{i=1}^{r_{\mathbf{t}}}$ множества $\mathscr{P} \mathbf{t}$, удовлетворяющую условиям предыдущего утверждения. Пусть $\left\{h_{i}^{\mathbf{t}_{1}}\right\}_{i=1}^{r_{\mathbf{t}_{1}}},\left\{h_{i}^{\mathbf{t}_{2}}\right\}_{i=1}^{r_{\mathbf{t}_{2}}}$ - две такие базы, причем $\mathbf{t}_{1} \neq \mathbf{t}_{2}$.

ЛЕмма 2.7. При любых $i=1, \ldots, r_{\mathbf{t}_{1}}, j=1, \ldots, r_{\mathbf{t}_{2}}$ нормы әлементов $h_{i}^{\mathbf{t}_{1}} u h_{j}^{\mathbf{t}_{2}}$ различны. 
ДокАЗАТЕЛЬСТво. Предположим, что для некоторых $i, j$ нормы элементов $h_{i}^{\mathbf{t}_{1}}, h_{j}^{\mathbf{t}_{2}}$ равны. Пусть для определенности $\mathbf{t}_{1} \prec \mathbf{t}_{2}$. Тогда по лемме 2.2 существует такое ненулевое $\beta \in k$, что $\eta\left(\beta h_{i}^{\mathbf{t}_{1}}+h_{j}^{\mathbf{t}_{2}}\right) \prec \eta\left(h_{j}^{\mathbf{t}_{2}}\right)$.

Элемент $h=\beta h_{i}^{\mathbf{t}_{1}}+h_{j}^{\mathbf{t}_{2}}$ лежит в $\mathscr{P}_{\mathbf{t}_{2}}$. В самом деле, в силу утверждения 2 леммы 2.3 выполняется равенство $\rho(h)=\rho\left(\beta h_{i}^{\mathbf{t}_{1}}+h_{j}^{\mathbf{t}_{2}}\right)=\rho\left(h_{j}^{\mathbf{t}_{2}}\right)=\mathbf{t}_{2}$, поскольку $\rho\left(h_{i}^{\mathbf{t}_{1}}\right)=\mathbf{t}_{1} \prec$ $\mathbf{t}_{2}=\rho\left(h_{j}^{\mathbf{t}_{2}}\right)$.

Кроме того, $\nu_{\mathbf{t}_{2}}(h)=\nu_{\mathbf{t}_{2}}\left(h_{j}^{\mathbf{t}_{2}}\right)$, т.е. свойство $(* *)$ в базисе $\left\{h_{i}^{\mathbf{t}_{2}}\right\}_{i=1}^{r_{\mathbf{t}_{2}}}$ не вьполнено, что неверно.

Совокупность баз $\left\{h_{i}^{\mathbf{t}}\right\}_{i=1}^{r_{\mathbf{t}}}$ для всех $\mathbf{t} \in \mathbb{N}_{0}^{n}$ назовем базой множества $\mathscr{P}$. Будем обозначать эту совокупность $\mathscr{B}$. Предыдущая лемма, таким образом, утверждает, что все элементы базы $\mathscr{B}$ имеют различные нормы.

УТВЕРЖДЕНИЕ 2.8. Всякий әлемент из ЯР может быть представлен в виде линейной комбиначии әлементов базы $\mathscr{B}$.

ДокАЗАтЕльство. Рассмотрим произвольный элемент $p$ множества $\mathscr{P}$. Будем вести доказательство индукцией по полунорме $p$.

Пусть $\rho(p)=\mathbf{t}$. По предположению индукции все элементы $\mathscr{P}$ с полунормой, меньшей $\mathbf{t}$, представимы в виде линейной комбинации элементов базы $\mathscr{B}$.

Поскольку система $\left\{h_{i}^{\mathbf{t}}\right\}_{i=1}^{r_{\mathbf{t}}}$ является базой для $\mathscr{P}_{\mathbf{t}}$, то

$$
\nu_{\mathbf{t}}(p)=\sum_{i=1}^{r_{\mathbf{t}}} \alpha_{i} \nu_{\mathbf{t}}\left(h_{i}^{\mathbf{t}}\right)=\nu_{\mathbf{t}}\left(\sum_{i=1}^{r_{\mathbf{t}}} \alpha_{i} h_{i}^{\mathbf{t}}\right)
$$

Поэтому $\rho\left(p-\sum_{i=1}^{r_{\mathbf{t}}} \alpha_{i} h_{i}^{\mathbf{t}}\right) \prec \rho(p)$. Используя индуктивное предположение, получаем искомьй результат.

Утверждение 2.8 и то свойство, что нормы различных элементов базы $\mathscr{B}$ различны, дают такое простое

СлЕДСТВИЕ 2.9. Норма всякого әлемента Я совпадает с нормой некоторого әлемента базы $\mathscr{B}$.

Обозначим через $\mathscr{N}$ множество всех норм элементов $\mathscr{P}$. В силу следствия 2.9 и того, что $\mathscr{B} \subset \mathscr{P}, \mathscr{N}$ совпадает с множеством всех норм элементов базы $\mathscr{B}$.

Пусть множество $\mathscr{N}$ порождает модуль $\mathscr{M} \subset \mathbb{Z}^{n}$ ранга $m$.

Теорема 2.10. Всякая система из $m+1$ әлементов $p_{1}, \ldots, p_{m+1} \in \mathscr{P}$ алгебраически зависима. Именно, существует многочлен $F\left(x_{1}, \ldots, x_{m+1}\right) \in k\left[x_{1}, \ldots, x_{m+1}\right]$ такой, что $F\left(p_{1}, \ldots, p_{m+1}\right)=0$.

ДокАЗАТЕЛЬСтво. Для произвольного $\mathbf{d} \in \mathbb{N}_{0}^{n}$ рассмотрим множество $\psi_{\mathbf{d}}$ всевозможных произведений $\pi=p_{1}^{l_{1}} \ldots p_{m+1}^{l_{m+1}}$ с неотрицательньми степенями $l_{i}$, удовлетворяющих условию

$$
\sum_{i=1}^{m+1} l_{i} \rho\left(p_{i}\right) \preceq \mathbf{d} .
$$


Множество $\psi_{\mathbf{d}}$ лежит в $\mathscr{P}$, поэтому всякий его элемент может быть представлен в виде некоторой линейной комбинации элементов базы $\mathscr{B}$ :

$$
\pi=\sum_{\substack{|\eta(h)| \preceq \rho(\pi), h \in \mathscr{B}}} \beta_{h} h .
$$

Рассмотрим множество $\phi_{\mathbf{d}}$ всех элементов $h \in \mathscr{B}$, входящих в линейное разложение какого-либо элемента $\pi \in \psi_{\mathbf{d}}$.

Если $h \in \phi_{\mathbf{d}}$, то

$$
|\eta(h)| \preceq \rho(\pi) \preceq \sum_{i=1}^{m+1} l_{i} \rho\left(p_{i}\right) \preceq \mathbf{d} .
$$

Заметим, что мощность пересечения модуля $\mathscr{M}$ с множеством элементов $\mathbb{Z}^{n}$, абсолютные значения которых ограничены сверху элементом $\mathbf{d}$, ограничивается сверху значениями многочлена $m$-й степени от $\sigma(\mathbf{d})$. Поскольку $\eta\left(\phi_{\mathbf{d}}\right)$ является подмножеством этого пересечения, и нормы всех элементов $\phi_{\mathbf{d}}$ различны, то

$$
\operatorname{card} \phi_{\mathbf{d}}=\operatorname{card} \eta\left(\phi_{\mathbf{d}}\right) \leqslant P_{m}(\sigma(\mathbf{d}))
$$

где $P_{m}(x)$ - многочлен $m$-й степени от $x$ с действительными коэффициентами.

Теперь оценим снизу мошность $\psi_{\mathbf{d}_{s}}$, где $\mathbf{d}_{s}=s \sum_{i=1}^{m+1} \rho\left(p_{i}\right)$. Все кортежи $\left(l_{1}, \ldots, l_{m+1}\right) \in \mathbb{N}_{0}^{m+1}$, у которых $l_{i} \leqslant s$ для любого $i=1, \ldots, m+1$, удовлетворяют условию $\sum_{i=1}^{m+1} l_{i} \rho\left(p_{i}\right) \preceq \mathbf{d}$. Количество таких кортежей

$$
s^{m+1}=\left(\frac{\sigma\left(\mathbf{d}_{s}\right)}{\sum_{i=1}^{m+1} \sigma\left(\rho\left(p_{i}\right)\right)}\right)^{m+1}=P_{m+1}\left(\sigma\left(\mathbf{d}_{s}\right)\right),
$$

т.е. мощность множества $\psi_{\mathbf{d}_{s}}$ ограничена снизу значениями многочлена $(m+1)$-й степени от $\sigma\left(\mathbf{d}_{s}\right)$ :

$$
\operatorname{card} \psi_{\mathbf{d}_{s}} \geqslant P_{m+1}\left(\sigma\left(\mathbf{d}_{s}\right)\right),
$$

где $P_{m+1}(x)$ - многочлен $(m+1)$-й степени от $x$ с действительными коэффициентами.

Поэтому, для некоторого $\mathbf{d}_{s}$ вьполняется оценка

$$
\operatorname{card} \phi_{\mathbf{d}_{s}} \leqslant P_{m}\left(\sigma\left(\mathbf{d}_{s}\right)\right)<P_{m+1}\left(\sigma\left(\mathbf{d}_{s}\right)\right) \leqslant \operatorname{card} \psi_{\mathbf{d}_{s}} .
$$

Это означает, что элементы множества $\psi_{\mathbf{d}_{s}}$ линейно зависимы, поскольку являются линейньми комбинациями символов $h \in \phi_{\mathbf{d}_{s}}$ в количестве, меньшем мошности $\psi_{\mathbf{d}_{s}}$.

Итак, мы нашли равную нулю линейную комбинацию произведений элементов $p_{1}, \ldots, p_{m+1}$ вида $p_{1}^{l_{1}} \ldots p_{m+1}^{l_{m+1}}$, где степени $l_{i}$ неотрицательны, что и требовалось.

Пусть заданы попарно коммутируюшие, алгебраически независимые элементы $g_{1}, \ldots, g_{m}$ алгебры $\mathscr{A}$.

Рассмотрим множество

$$
\mathscr{P}=\left\{p\left(g_{1}, g_{2}, \ldots, g_{m}\right) \mid p \in k\left[x_{1}, x_{2}, \ldots, x_{m}\right], m \in \mathbb{N}_{0}\right\}
$$

полиномов от этих элементов.

Это множество, очевидно, является подалгеброй $\mathscr{A}$. Поэтому к нему применима теорема 2.10. 
УТВЕРЖДЕНИЕ 2.11. Существует система $p_{1}, \ldots, p_{m}$ многочленов от $g_{i}, i=$ $1, \ldots, m$, нормы которых образуют линейно независимую систему.

ДокАЗАТЕЛЬСТВо. Из теоремы 2.10 и алгебраической независимости элементов $g_{1}, \ldots, g_{m}$ следует, что ранг модуля $\mathscr{M}$, порожденного нормами элементов $\mathscr{P}$, не может быть меньше $m$. То есть существует искомая система многочленов $p_{1}, \ldots, p_{m}$, нормы которых образуют линейно независимую систему.

ОПРЕДЕЛЕНИЕ 2.12. Пусть элементы $b_{1}, b_{2}$ базиса $\mathfrak{B}$ таковы, что $\eta\left(\left[b_{1}, b_{2}\right]\right) \prec \eta\left(b_{1}\right)+$ $\eta\left(b_{2}\right)$. Будем называть базисные элементы, обладающие указанным свойством, $n c e в д о-$ коммутирующ,ими.

ЛЕмма 2.13. Пусть два әлемента $f, g \in \mathscr{A}$ снормами $\eta(f)=\mathbf{t}_{1} u \eta(g)=\mathbf{t}_{2}$ коммутируют между собой. Тогда базисные әлементы $b_{1}, b_{2}$ такие, что $\eta\left(b_{1}\right)=$ $\mathbf{t}_{1}$ и $\eta\left(b_{2}\right)=\mathbf{t}_{2}$, являются псевдокоммутируюшими.

ДокАЗАТЕЛЬСТво. Пусть $f=f^{\prime}+\alpha b_{1}, g=g^{\prime}+\beta b_{2}$, где $\eta\left(f^{\prime}\right) \prec \eta(f)$ и $\eta\left(g^{\prime}\right) \prec \eta(g)$. По условию $f g=\left(f^{\prime}+\alpha b_{1}\right)\left(g^{\prime}+\beta b_{2}\right)=\left(g^{\prime}+\beta b_{2}\right)\left(f^{\prime}+\alpha b_{1}\right)=g f$, т.е. $\alpha \beta\left(b_{1} b_{2}-\right.$ $\left.b_{2} b_{1}\right)=\left[g^{\prime}, f^{\prime}\right]+\left[g^{\prime}, \alpha b_{1}\right]+\left[\beta b_{2}, f^{\prime}\right]$. Из свойств N1)-N3) нормы получаем

$$
\eta\left(\left[b_{1}, b_{2}\right]\right)=\eta\left(\left[g^{\prime}, f^{\prime}\right]+\left[g^{\prime}, \alpha b_{1}\right]+\left[\beta b_{2}, f^{\prime}\right]\right) \prec \eta(f)+\eta(g)=\eta\left(b_{1}\right)+\eta\left(b_{2}\right) .
$$

ОПрЕДЕЛЕниЕ 2.14. Элементы базиса $\mathfrak{B}$, имеющие линейно независимые нормы, будем назьвать независимыми.

ТЕОрема 2.15. Максимальное количество $N$ алгебраически независимых коммутирующих друг с другом әлементов алгебры $\mathscr{A}$ не превышает максимального количества $M$ независимых, псевдокоммутирующих друг с другом әлементов базиса $\mathfrak{B}$.

ДокАЗАТЕЛЬСТво. Пусть $g_{1}, \ldots, g_{N}$ - максимальная алгебраически независимая система коммутирующих элементов алгебры $\mathscr{A}$.

По утверждению 2.11 существует система $p_{1}, \ldots, p_{N}$ многочленов от элементов $g_{1}, \ldots, g_{N}$ такая, что нормы $p_{1}, \ldots, p_{N}$ линейно независимы.

Многочлены $p_{1}, \ldots, p_{N}$ попарно коммутируют, так как $g_{1}, \ldots, g_{N}$ система коммутирующих элементов. Поэтому базисные элементы $b_{1}, \ldots, b_{N}$, где $\eta\left(b_{1}\right)=\eta\left(p_{1}\right), \ldots$, $\eta\left(b_{N}\right)=\eta\left(p_{N}\right)$, по лемме 2.13 являются попарно псевдокоммутирующими и имеют линейно независимые нормы.

\section{3. Примеры}

Алгебра квантовых лорановских многочленов. Пусть задана матрица $Q=$ $\left(q_{i j}\right) \in \operatorname{Mat}\left(n, k^{*}\right)$, где $n \geqslant 2$, со следующими свойствами: $q_{i i}=1, q_{i j}=q_{j i}^{-1}$.

Алгеброй квантовых лорановских многочленов над полем $k$ будем назьвать ассоциативную алгебру

$$
\mathscr{L}=k_{Q}\left[X_{1}^{ \pm 1}, \ldots, X_{n}^{ \pm 1}\right]
$$

над $k$, порожденную $2 n$ элементами $X_{1}^{ \pm 1}, \ldots, X_{n}^{ \pm 1}$, с определяюшими соотношениями:

$$
\begin{aligned}
X_{i} X_{i}^{-1} & =X_{i}^{-1} X_{i}=1, & & 1 \leqslant i \leqslant n, \\
X_{i} X_{j} & =q_{i j} X_{j} X_{i}, & & 1 \leqslant i, j \leqslant n .
\end{aligned}
$$


Из определяющих соотношений алгебры $\mathscr{L}$ видно, что всякий элемент $X_{i_{1}}^{\varepsilon_{1}} \ldots X_{i_{r}}^{\varepsilon_{r}}$, где все $\varepsilon_{i}= \pm 1$, однозначно представим в виде $\alpha X_{1}^{t_{1}} \ldots X_{n}^{t_{n}}$ при некоторых целых $t_{1}, \ldots, t_{n}$ и $\alpha \in k^{*}$. Поэтому всякий элемент $f$ алгебры $\mathscr{L}$ однозначно представляется в виде линейной комбинации элементов вида $X_{1}^{t_{1}} \ldots X_{n}^{t_{n}}$, т.е. множество $\left\{X_{1}^{t_{1}} \ldots X_{n}^{t_{n}} \mid\right.$ $\left.\left(t_{1}, \ldots, t_{n}\right) \in \mathbb{Z}^{n}\right\}$ является базисом алгебры $\mathscr{L}$. В дальнейшем, элементы этого базиса будем назьвать мономами и для краткости писать $X^{\mathbf{t}}$ вместо $X_{1}^{t_{1}} \ldots X_{n}^{t_{n}}$. Кортеж $\mathbf{t}=\left(t_{1}, \ldots, t_{n}\right)$ будем назьвать степенью монома $X^{\mathbf{t}}$.

Введем нормирование на $\mathscr{L}$, связанное с порядком $\succ$. Для ненулевого многочлена $f$ норма $\|f\|_{\mathscr{L}}$ - это кортеж из $\mathbb{Z}^{n}$, являюшийся максимальным (относительно $\left.\succ\right)$ в множестве степеней мономов, входящих в $f$.

УТВЕРЖДЕНИЕ 3.1. Нормирование $\|\cdot\|_{\mathscr{L}}$ обладает следующими свойствами:

1) $\|f g\|_{\mathscr{L}}=\|f\|_{\mathscr{L}}+\|g\|_{\mathscr{L}}$,

2) $\|f+g\|_{\mathscr{L}} \preceq \max \left\{\|f\|_{\mathscr{L}},\|g\|_{\mathscr{L}}\right\}$.

ДокАЗАТЕЛЬСтво. 1) Любой моном из разложения $f g$ является произведением монома $m_{1}$ из разложения $f$ и монома $m_{2}$ из разложения $g$. Поэтому степень монома из разложения $f g$ - это сумма степеней $\left\|m_{1}\right\|_{\mathscr{L}}=\mathbf{t}_{1}$ и $\left\|m_{2}\right\|_{\mathscr{L}}=\mathbf{t}_{2}$. В силу свойств 1$\left.), 2\right)$ порядка $\succ$ моном максимальной степени из разложения $f g$ - это произведение монома максимальной степени из разложения $f$ и монома максимальной степени из разложения $g$.

2) Мономы, входящие в разложение суммы $f+g$-это либо мономы из разложения $f$, либо из разложения $g$.

Из утверждения 3.1 и определения нормирования $\|\cdot\|_{\mathscr{L}}$ ясно, что оно удовлетворяет всем свойствам нормы $\eta$ из предыдущего раздела, если в качестве базиса $\mathfrak{B}$ выбрать мономиальный базис.

Поэтому мы можем определить для $\|\cdot\|_{\mathscr{L}}$ соответствуюшее отображение $\rho \mathscr{L}$. Для ненулевого многочлена $f$ значение отображения $\rho \mathscr{L}$ - это кортеж из $\mathbb{N}_{0}^{n}$, являющийся максимальным (относительно $\succ$ ) в множестве абсолютных значений степеней мономов, входящих в $f$.

УТВЕРЖДЕНИЕ 3.2. Отображсение $\rho \mathscr{L}$ удовлетворяет условию

$$
\rho \mathscr{L}(f g) \preceq \rho \mathscr{L}(f)+\rho \mathscr{L}(g),
$$

т.е. является полунормой, согласованной с нормой $\|\cdot\| \mathscr{L}$.

ДокАЗАТЕЛЬСтво. Любой моном из $f g$ является произведением монома $m_{1}$ из $f$ и монома $m_{2}$ из $g$. Поэтому степень монома из $f g$-это сумма степеней $\mathbf{t}_{1}$ и $\mathbf{t}_{2}$. По утверждению 1.4 имеет место оценка $\left|\mathbf{t}_{1}+\mathbf{t}_{2}\right| \preceq\left|\mathbf{t}_{1}\right|+\left|\mathbf{t}_{2}\right|$. Поэтому

$$
\max _{\mathbf{t}_{1}, \mathbf{t}_{2}}\left\{\left|\mathbf{t}_{1}+\mathbf{t}_{2}\right|\right\} \preceq \max _{\mathbf{t}_{1}}\left\{\left|\mathbf{t}_{1}\right|\right\}+\max _{\mathbf{t}_{2}}\left\{\left|\mathbf{t}_{2}\right|\right\} \text {. }
$$

Итак, алгебра $\mathscr{L}$ удовлетворяет всем требованиям раздела 2 , т.е. для нее вьполнена теорема 2.10 .

ЛЕмма 3.3. Псевдокоммутирующие мономы $X^{\mathbf{t}_{1}}, X^{\mathbf{t}_{2}}$ коммутируют друг с другом. 
ДокАЗАТЕЛЬСТвО. По условию $\left\|\left[X^{\mathbf{t}_{1}}, X^{\mathbf{t}_{2}}\right]\right\|_{\mathscr{L}} \prec \mathbf{t}_{1}+\mathbf{t}_{2}$. Поскольку $X^{\mathbf{t}_{1}} X^{\mathbf{t}_{2}}=$ $\alpha X^{\mathbf{t}_{1}+\mathbf{t}_{2}}$ и $X^{\mathbf{t}_{2}} X^{\mathbf{t}_{1}}=\beta X^{\mathbf{t}_{1}+\mathbf{t}_{2}}$, для коммутанта получаем $\left[X^{\mathbf{t}_{1}}, X^{\mathbf{t}_{2}}\right]=(\alpha-\beta) X^{\mathbf{t}_{1}+\mathbf{t}_{2}}$, откуда $\alpha-\beta=0$, т.е. $\left[X^{\mathbf{t}_{1}}, X^{\mathbf{t}_{2}}\right]=0$.

В силу леммы 3.3 теорема 2.15 в случае алгебры $\mathscr{L}$ принимает следующий вид.

Теорема 3.4. Максимальное количество $N$ алгебраически независимых коммутирующих друг с другом әлементов алгебры $\mathscr{L}$ совпадает с максимальным количеством $M$ независимых коммутирующих друг с другом мономов из $\mathscr{L}$.

ДокАЗАтЕЛЬСтво. Очевидно, $N \geqslant M$. С другой стороны, по теореме $2.15 N \leqslant$ $M$, поскольку по лемме 3.3 всякая система псевдокоммутирующих мономов является системой коммутируюших мономов.

ЛЕмма 3.5. Попарно коммутирующие мономы, имеющие линейно независимые нормы, алгебраически независимы.

ДокАЗАТЕЛЬСтво. Пусть мономы $X^{\mathbf{d}_{1}}, \ldots, X^{\mathbf{d}_{r}}$ удовлетворяют условию леммы.

Если бы существовал такой многочлен $F\left(x_{1}, \ldots, x_{r}\right) \in k\left[x_{1}, \ldots, x_{r}\right]$, что $F\left(X^{\mathbf{d}_{1}}, \ldots, X^{\mathbf{d}_{r}}\right)=0$, то произведения вида $\left(X^{\mathbf{d}_{1}}\right)^{i_{1}} \ldots\left(X^{\mathbf{d}_{r}}\right)^{i_{r}}$, входящие в выражение $F\left(X^{\mathbf{d}_{1}}, \ldots, X^{\mathbf{d}_{r}}\right)$ должны были бы сокращаться друг с другом. Но равенство

$$
\left(X^{\mathbf{d}_{1}}\right)^{i_{1}} \ldots\left(X^{\mathbf{d}_{r}}\right)^{i_{r}}=\left(X^{\mathbf{d}_{1}}\right)^{j_{1}} \ldots\left(X^{\mathbf{d}_{r}}\right)^{j_{r}}
$$

в силу линейной независимости $\mathbf{d}_{1}, \ldots, \mathbf{d}_{r}$ равносильно равенству $\left(i_{1}, \ldots, i_{r}\right)=$ $\left(j_{1}, \ldots, j_{r}\right)$, поэтому многочлен $F$ должен быть тождественным нулем.

В силу леммы 3.5 теорему 3.4 можно переформулировать следующим образом.

Теорема 3.6. Максимальное количество $N$ алгебраически независимых коммутирующих друг с другом многочленов из кольиа $\mathscr{L}$ совпадает с максимальным количеством $M$ алгебраически независимых коммутирующих друг с другом мономов из $\mathscr{L}$.

Бруксом в работе [8] показано, что мощность максимальной системы независимых попарно коммутирующих элементов мономиального базиса алгебры $\mathscr{L}$ совпадает с размерностью Крулля этой алгебры. Из этого результата и предыдущей теоремы вытекает

СлЕДСТВИЕ 3.7. Максимальное количество $N$ алгебраически независимых коммутирующих друг с другом многочленов из алгебры $\mathscr{L}$ совпадает с размерностью Крулля әтой алгебры.

Квантовые многочлены, квантовый аналог алгебр Вейля и универсальные кодействующие. Еще один пример алгебры, обладающей нормой и согласованной с ней полунормой, дает следующая конструкция.

Пусть матрища $Q=\left(q_{i j}\right) \in \operatorname{Mat}\left(n, k^{*}\right)$ такая же, как в предыдущем пункте.

Рассмотрим ассоциативную алгебру $\mathfrak{A}_{n}^{Q, \alpha}$ над полем $k$ с порождающими $X_{1}, \ldots, X_{n}$ и со следующими определяющими соотношениями:

$$
X_{i} X_{j}=q_{i j} X_{j} X_{i}+\sum_{\mathbf{t} \prec \delta\left(X_{i} X_{j}\right)} \alpha_{\mathbf{t}}^{i j} X^{\mathbf{t}}, \quad 1 \leqslant i<j \leqslant n .
$$

Здесь, как и раньше, символ $X^{\mathbf{t}}$ означает произведение $X_{1}^{t_{1}} \ldots X_{n}^{t_{n}}$, причем $\mathbf{t}=$ $\left(t_{1}, \ldots, t_{n}\right) \in \mathbb{N}_{0}^{n}$, а $\delta\left(X^{\mathbf{t}}\right)$ равно $\mathbf{t}$. 
ОПРЕДЕЛЕниЕ 3.8. Пусть $X_{i_{1}} \ldots X_{i_{r}}$ - некоторое произведение образующих $X_{1}, \ldots, X_{n}$. Если переставить символы в последовательности $X_{i_{1}} \ldots X_{i_{r}}$ так, чтобы индексы не убьвали, мы получим некоторый моном $X^{\mathbf{t}}$. Кортеж $\mathbf{t}$ будем назьвать $c m e-$ пенъю произведения $X_{i_{1}} \ldots X_{i_{r}}$.

Таким образом, использованное в определении алгебры $\mathfrak{A}_{n}^{Q, \alpha}$ отображение $\delta$ сопоставляет моному $X^{\mathbf{t}}$ его степень $\mathbf{t}$.

ЛЕмма 3.9. Всякое произведение $X_{i_{1}} \ldots X_{i_{r}}$ в алгебре $\mathfrak{A}_{n}^{Q, \alpha}$ представимо в виде

$$
X_{i_{1}} \ldots X_{i_{r}}=\gamma X^{\mathbf{t}}+\sum_{\mathbf{d} \prec \mathbf{t}} \beta_{\mathbf{d}} X^{\mathbf{d}}, \quad \gamma \in k^{*}, \quad \beta_{\mathbf{d}} \in k,
$$

әде $X^{\mathbf{t}}$-моном, получающийся из произведения $X_{i_{1}} \ldots X_{i_{r}}$ перестановкой порождающих $X_{i}$ в порядке неубивания индексов.

ДокАЗАТЕЛЬСТво. Каждому произведению $X_{i_{1}} \ldots X_{i_{r}}$ мы можем сопоставить его степень $\mathbf{t}$ указанным выше способом. Кроме того, мы можем сопоставить этому произведению количество “беспорядков", т.е. пар индексов $\left(i_{j_{1}}, i_{j_{2}}\right), 1 \leqslant j_{1}, j_{2} \leqslant r$, для которых $i_{j_{1}}>i_{j_{2}}$, но $j_{1}<j_{2}$.

Для произведений, где $r=2$, требуемые соотношения - это просто определяющие соотношения алгебры $\mathfrak{A}_{n}^{Q, \alpha}$.

Рассмотрим произвольное произведение $\chi=X_{i_{1}} \ldots X_{i_{r}}$. Предположим, что утверждение леммы доказано для произведений, у которых степень меньше (относительно порядка $\prec)$ степени $\chi$, и для произведений, у которых степень равна степени $\chi$, но количество беспорядков меньше, чем у $\chi$.

Пусть $i_{j}, i_{j+1}-$ соседние индексы, образующие беспорядок в $\chi$. Тогда

$$
\begin{aligned}
& X_{i_{1}} \ldots X_{i_{r}}=X_{i_{1}} \ldots X_{i_{j-1}}\left(q_{i_{j+1} i_{j}}^{-1} X_{i_{j+1}} X_{i_{j}}+\sum_{\mathbf{t} \prec \eta\left(X_{i_{j+1}} X_{i_{j}}\right)} \alpha_{\mathbf{t}}^{i j} X^{\mathbf{t}}\right) X_{i_{j+2}} \ldots X_{i_{r}} \\
& =q_{i_{j+1} i_{j}}^{-1} X_{i_{1}} \ldots X_{i_{j+1}} X_{i_{j}} \ldots X_{i_{r}}+\sum_{\mathbf{t} \prec \eta\left(X_{i_{j+1}} X_{i_{j}}\right)} \alpha_{\mathbf{t}}^{i j} X_{i_{1}} \ldots X_{i_{j-1}} X^{\mathbf{t}} X_{i_{j+2}} \ldots X_{i_{r}} .
\end{aligned}
$$

Теперь заметим, что у произведения $X_{i_{1}} \ldots X_{i_{j+1}} X_{i_{j}} \ldots X_{i_{r}}$ количество беспорядков меньше, чем у $\chi$, а у произведений $X_{i_{1}} \ldots X_{i_{j-1}} X^{\mathbf{t}} X_{i_{j+2}} \ldots X_{i_{r}}$ степень меньше степени $\chi$, т.е. для них утверждение леммы верно. Записывая для этих произведений соответствующие соотношения, получаем искомый результат.

ЛЕмма 3.10. Мономы $\left\{X^{\mathbf{t}} \mid \mathbf{t}=\left(t_{1}, \ldots, t_{n}\right) \in \mathbb{N}_{0}^{n}\right\}$ образуют линейно независимую систему в алгебре $\mathfrak{A}_{n}^{Q, \alpha}$.

ДокАЗАТЕЛЬСтво. Предположим, что $\sum_{i=1}^{r} \beta_{i} X^{\mathbf{t}_{i}}=0$. Не ограничивая общности, мы можем считать, что $\mathbf{t}_{1} \prec \cdots \prec \mathbf{t}_{r}$.

Из леммы 3.9 следует, что всякое представление монома $X^{\mathbf{t}}$ в алгебре $\mathfrak{A}_{n}^{Q, \alpha}$ обязательно содержит произведение $X_{i_{1}} \ldots X_{i_{r}}$, степень которого равна $\mathbf{t}$. Поэтому всякое представление суммы $\sum_{i=1}^{r} \beta_{i} X^{\mathbf{t}_{i}}$ содержит некоторое произведение $X_{i_{1}} \ldots X_{i_{r}}$ степени $\mathbf{t}_{r} \mathbf{c}$ ненулевьм коэффициентом, так как другие мономы не могут дать слагаемое степени, бо́льшей их собственной степени. Следовательно, указанное равенство возможно лишь при всех $\beta_{i}$ равных нулю. 
Из лемм 3.9, 3.10 следует, что множество мономов $\left\{X^{\mathbf{t}} \mid \mathbf{t} \in \mathbb{N}_{0}^{n}\right\}$ образует базис алгебры $\mathfrak{A}_{n}^{Q, \alpha}$ как векторного пространства над $k$.

Пусть $f=\sum_{i=1}^{r} \beta_{i} X^{\mathbf{t}_{i}}$ - произвольньй ненулевой элемент алгебры $\mathfrak{A}_{n}^{Q, \alpha}$. Положим $\delta(f)=\max \left\{\mathbf{t}_{i} \mid 1 \leqslant i \leqslant r\right\}$, здесь максимум берется относительно порядка $\prec$.

УТВЕРЖДЕНИЕ 3.11. Отображсение $\delta$ обладает следующими свойствами:

1) $\delta(f g)=\delta(f)+\delta(g)$;

2) $\delta(f+g) \preceq \max \{\delta(f), \delta(g)\}$.

ДокАЗАТЕЛЬСТво аналогично доказательству леммы 3.1.

Из доказанных лемм следует, что $\delta$ обладает всеми свойствами нормы $\eta$ из раздела 2 , если в качестве базиса $\mathfrak{B}$ взять базис $\left\{X^{\mathbf{t}} \mid \mathbf{t} \in \mathbb{N}_{0}^{n}\right\}$. Кроме того, соответствующее отображение $\rho$ совпадает с $\delta$, поскольку все значения $\delta$ лежат в $\mathbb{N}_{0}^{n}$, т.е. в силу утверждения 1) леммы $3.11 \rho \equiv \delta$ является полунормой, согласованной с нормой $\delta$.

Итак, для алгебры $\mathfrak{A}_{n}^{Q, \alpha}$ верны все результаты раздела 2.

Теперь рассмотрим несколько примеров алгебр $\mathfrak{A}_{n}^{Q, \alpha}$.

ПримеР 1. При всех $\alpha_{\mathbf{t}}^{i j}$, равных нулю, получаем алгебру с соотношениями

$$
X_{i} X_{j}=q_{i j} X_{j} X_{i}, \quad 1 \leqslant i<j \leqslant n,
$$

- хорошо известную алгебру квантовых многочленов $k_{Q}\left[X_{1}, \ldots, X_{n}\right]$.

ПримеР 2. Алев и Дюма в работе [5] рассмотрели следующий квантовый аналог алгебр Вейля.

Пусть $\bar{q}=\left(q_{1}, \ldots, q_{n}\right)$ - кортеж ненулевых элементов поля $k$ и $\Lambda$ - матрища со свойствами $\lambda_{i i}=1, \lambda_{i j}=\lambda_{j i}^{-1}$. Положим $\mu_{i j}=\lambda_{i j} q_{i}$ при $1 \leqslant i<j \leqslant n$. По определению квантовой алгеброй Вейля $A_{n}^{\bar{q}, \Lambda}(k)$ назьвается ассоциативная алгебра над полем $k$ c $2 n$ порождаюшими $X_{1}, \ldots, X_{n}, Y_{1}, \ldots, Y_{n}$ и с определяющими соотношениями

$$
\begin{aligned}
X_{i} X_{j} & =\mu_{i j} X_{j} X_{i}, \\
Y_{i} Y_{j} & =\lambda_{i j} Y_{j} Y_{i}, \quad 1 \leqslant i<j \leqslant n, \\
X_{i} Y_{j} & =\lambda_{j i} Y_{j} X_{i}, \\
X_{j} Y_{i} & =\mu_{i j} Y_{i} X_{j}, \\
X_{j} Y_{j} & =1+q_{j} Y_{j} X_{j}+\sum_{1 \leqslant l<j}\left(q_{l}-1\right) Y_{l} X_{l}, \quad 1 \leqslant j \leqslant n .
\end{aligned}
$$

Нетрудно убедиться в том, что эта алгебра изоморфна алгебре $\mathfrak{A}_{2 n}^{\mathscr{Q}, \alpha}$, для некоторых $\mathscr{Q}, \alpha$.

ПримеР 3. Демидов в обзоре [6] рассматривает универсальные кодействующие на семейство $\mathscr{S}$ алгебры $M(\mathscr{S})$.

Пусть семейство $\mathscr{S}$ состоит из двух алгебр:

$$
\begin{aligned}
& A_{Q}=k\left\langle x_{1}, \ldots, x_{n}\right\rangle /\left(x_{i} x_{j}-q_{i j}^{-1} x_{j} x_{i}\right), \\
& B_{P}=k\left\langle\xi_{1}, \ldots, \xi_{n}\right\rangle /\left(\xi_{i} \xi_{j}+p_{i j} \xi_{j} \xi_{i}, \xi_{i}^{2}\right)
\end{aligned}
$$


где $Q=\left(q_{i j}\right), P=\left(p_{i j}\right)$ - матрицы параметров, причем

$$
p_{i j} p_{j i}=q_{i j} q_{j i}=p_{i i}=q_{i i}=1 .
$$

Тогда алгебра $M(\mathscr{S})=M_{P, Q}$ оказьвается ассоциативной алгеброй над полем $k$ с $n^{2}$ порождающими $z_{i}^{j}, 1 \leqslant i, j \leqslant n$, и с определяющими соотношениями

$$
\begin{aligned}
z_{i}^{m} z_{j}^{m} & =q_{i j}^{-1} z_{j}^{m} z_{i}^{m}, \\
z_{i}^{m} z_{i}^{l} & =p_{m l}^{-1} z_{i}^{l} z_{i}^{m}, \\
z_{i}^{m} z_{j}^{l}-q_{i j}^{-1} q_{m l} z_{j}^{l} z_{i}^{m} & =-q_{m l} z_{i}^{l} z_{j}^{m}+q_{i j}^{-1} z_{j}^{m} z_{i}^{l}, \\
z_{i}^{m} z_{j}^{l}-p_{i j} p_{m l}^{-1} z_{j}^{l} z_{i}^{m} & =p_{m l}^{-1} z_{i}^{l} z_{j}^{m}-p_{i j} z_{j}^{m} z_{i}^{l},
\end{aligned}
$$

для всех $1 \leqslant i<j \leqslant n$ и $1 \leqslant m<l \leqslant n$.

УТВЕРЖДЕНИЕ 3.12. Пусть параметры $p_{i j}, q_{i j}$ удовлетворяют соотношению $p_{i j} q_{i j}=c^{\operatorname{sgn}(j-i)}$ для некоторого $c \in k^{*}, c \neq-1$. Тогда алгебра $M_{P, Q}=M_{P, Q, c}$ изоморфна алгебре $\mathfrak{A}_{n^{2}}^{\mathscr{Q}, \alpha}$ для некоторых $\mathscr{Q}, \alpha$.

ДокАЗАТЕЛЬСтво. По условию $p_{i j}=c^{\operatorname{sgn}(j-i)} q_{i j}^{-1}$. Перепишем соотношения следуюшим образом:

$z_{i}^{m} z_{j}^{l}-p_{i j} p_{m l}^{-1} z_{j}^{l} z_{i}^{m}-p_{m l}^{-1} z_{i}^{l} z_{j}^{m}+p_{i j} z_{j}^{m} z_{i}^{l}=z_{i}^{m} z_{j}^{l}-q_{i j}^{-1} q_{m l} z_{j}^{l} z_{i}^{m}-q_{m l} c^{-1} z_{i}^{l} z_{j}^{m}+q_{i j}^{-1} c z_{j}^{m} z_{i}^{l}$.

Отсюда

$$
q_{m l} z_{i}^{l} z_{j}^{m}-q_{i j}^{-1} z_{j}^{m} z_{i}^{l}=-q_{m l} c^{-1} z_{i}^{l} z_{j}^{m}+q_{i j}^{-1} c z_{j}^{m} z_{i}^{l}
$$

или

$$
q_{m l}\left(1+c^{-1}\right) z_{i}^{l} z_{j}^{m}=q_{i j}^{-1}(1+c) z_{j}^{m} z_{i}^{l} .
$$

При $c \neq-1$ имеем

$$
\begin{aligned}
& z_{i}^{l} z_{j}^{m}=q_{i j}^{-1} q_{m l}^{-1}(1+c)\left(1+c^{-1}\right)^{-1} z_{j}^{m} z_{i}^{l}=\lambda_{i l j m} z_{j}^{m} z_{i}^{l}, \\
& z_{i}^{m} z_{j}^{l}=q_{i j}^{-1} q_{m l} z_{j}^{l} z_{i}^{m}+\left(q_{m l} c^{-1}-q_{i j}^{-1} c\right) \lambda_{i l j m}^{-1} z_{i}^{l} z_{j}^{m} .
\end{aligned}
$$

Итак, все соотношения переписаны в виде

$$
\begin{aligned}
z_{i}^{m} z_{j}^{m} & =\lambda_{i m j m} z_{j}^{m} z_{i}^{m}, \\
z_{i}^{m} z_{i}^{l} & =\lambda_{i m i l} z_{i}^{l} z_{i}^{m}, \\
z_{i}^{l} z_{j}^{m} & =\lambda_{i l j m} z_{j}^{m} z_{i}^{l}, \\
z_{i}^{m} z_{j}^{l} & =\lambda_{i m j l} z_{j}^{l} z_{i}^{m}+\mu_{i m j l} z_{i}^{l} z_{j}^{m},
\end{aligned}
$$

при некоторых коэффициентах $\lambda_{i m j l}, \mu_{i m j l} \in k, \lambda_{i m j l} \neq 0$.

Положим $z_{i}^{m}=X_{(i-1) n+m}$. Нетрудно видеть, что все произведения $z_{i}^{m}$, входящие в определяюшие соотношения алгебры $M_{P, Q, c}$, являются мономами вида $X^{\mathbf{t}}$ и что для любой пары $X_{s}, X_{r}, 1 \leqslant s, r \leqslant n^{2}$, среди определяющих соотношений найдется соответствующее коммутационное соотношение. Остается проверить, что последнее соотношение является соотношением вида (1). Действительно, пусть $1 \leqslant i<j \leqslant n$ и $1 \leqslant m<l \leqslant n$. Тогда $(i-1) n+l>(i-1) n+m$, т.е.

$$
\delta\left(z_{i}^{l} z_{j}^{m}\right)=\delta\left(X_{(i-1) n+l} X_{(j-1) n+m}\right) \prec \delta\left(X_{(i-1) n+m} X_{(j-1) n+l}\right)=\delta\left(z_{i}^{m} z_{j}^{l}\right),
$$

что и требовалось. 


\section{СПИСОК ЦИТИРОВАННОЙ ЛИТЕРАТУРЫ}

[1] Artamonov V. A., Cohn P. M. The skew field of rational functions on the quantum plane // J. Math. Sci. 1999. V. 93. №6. P. 824-829.

[2] Cohn P. M. Centralisateur dans les corps libres // Ecole de Printemps d'Informatique Théorique, "Séries formelles en variables non commutatives et applications" / ed. J. Berstel: Vieux-Boucau les Bains (Landes), 1978. P. 45-54.

[3] Артамонов В. А. Тело квантовых рациональных функций // УМН. 1999. Т. 54. № 4. C. $151-152$.

[4] Зеленова С. А. Коммутативные подалгебры кольца квантовых многочленов и тела квантовых лорановских рядов // Матем. сб. 2001. Т. 192. № 3. С. 55-64.

[5] Alev J., Dumas F. Sur le corps des fractions de certaines algèbres quantiques // J. of Algebra. 1994. V. 170. №1. P. 229-265.

[6] Демидов Е. Е. О некоторых аспектах теории квантовых групп // УМН. 1993. Т. 48. №6. C. $39-74$.

[7] Brown K. A., Goodearl K. R. Lecture on algebraic quantum groups. Basel-Boston: Birkhäuser, 2002.

[8] Brookes C. J. B. Crossed products and finitely presented groups // J. Group Theory. 2000. V. 3. P. 433-444.

Институт системного программирования РАН

Поступило

08.07.2002 